\title{
CRITICALITY DEPENDENCE ON DATA AND PARAMETERS FOR A PROBLEM IN COMBUSTION THEORY
}

\author{
K. K. TAM ${ }^{1}$ \\ (Received 16 August 1983; revised 17 March 1985)
}

\begin{abstract}
A central problem in the theory of combustion, consisting of a nonlinear parabolic equation together with initial and boundary conditions, is considered. The influence of the initial and boundary data are examined. In the main part of the study, a two-step linearization is developed such that the interesting features of the original problem are given by the solution of a non-linear ordinary differential equation. Approximate solutions are obtained and upper and lower solutions are used to assess the validity of the approximations. Whenever possible, results are compared with those obtained previously and there is good agreement in all cases.
\end{abstract}

\section{Introduction}

A central problem in combustion theory is the initial and boundary value problem (IBVP) consisting of the nonlinear parabolic equation

$$
\frac{\partial \theta}{\partial t}=\nabla^{2} \theta+\delta f(\theta) ; \quad \mathbf{x} \in D, t>0,
$$

where $f(\theta)=\exp [\alpha \theta /(\alpha+\theta)]$, and the conditions

$$
\theta(\mathbf{x}, 0)=h(\mathbf{x}) \geqslant 0, \quad \mathbf{x} \in D,
$$

and

$$
\theta(\mathbf{x}, t)=g(\mathbf{x}, t) ; \quad \mathbf{x} \text { on } \partial D, t>0
$$

\footnotetext{
${ }^{1}$ Department of Mathematics and Statistics, McGill University, Montreal, Quebec, Canada H3A 2K6

(C) Copyright Australian Mathematical Society 1986, Serial-fee code 0334-2700/86
} 
Here, $\theta$ is the temperature, $\mathbf{x}$ and $t$ are the non-dimensionalized spatial and time variables, $\delta$ is a parameter incorporating the chemical properties of the combustible material, the temperature of assembly, as well as its geometrical dimensions, and $\alpha$ is a parameter related to the activation energy of the material occupying the region $D$. Typically, $\alpha$ is considerably greater than unity.

A number of investigators have studied this problem. The derivation of (1-1) and reference to the early work can be found in [4]. A usual simplification is the so-called "expanding the exponent", namely, the nonlinear term in $(1-1)$ is replaced by $\exp (\theta)$. The result is that the time-independent version of $(1-1)$ is reduced to the Liouville equation, which is an important equation used to describe a variety of physical phenomena, among them fluid mechanics and plasma physics. Exact solutions can be found for the Liouville equation for simple geometries, and the early analytical work on combustion theory is in this category. It is found that when $\delta$ exceeds a certain value, $\delta_{\mathrm{cr}}$, which is dependent on the geometry, the Liouville equation has no solution, which is interpreted to mean that ignition results. While the simpler equation gives useful information on $\delta_{\mathrm{cr}}$, it does have the disadvantage of losing track of the influence of $\alpha$ on $\delta_{\mathrm{cr}}$. More importantly, replacing the term $\exp [\alpha \theta /(\alpha+\theta)]$, which is bounded by $e^{\alpha}$ regardless of the value of $\theta$, by the term $\exp (\theta)$, which grows exponentially with $\theta$, changes the character of the nonlinear term. The IBVP (1-1), (1-2), (1-3), has for every $L$ a unique solution bounded for all $0<t<L$, [1], for all values of $\delta$. Such a result is not obtainable for the simplified equation without further restrictions.

In 1961, Parks [6] integrated numerically equations (1-1), (1-2), and (1-3), with $g(\mathbf{x}, t)=h(\mathbf{x})=0$, and obtained the critical values $\delta_{\mathrm{cr}}$, for a range of values of $\alpha$, for a number of simple geometrical shapes. In 1975, Sattinger [7] considered the stability of the multiple time-independent solutions corresponding to (1-1), (1-2), (1-3) with $h(\mathbf{x})=g(\mathbf{x}, t)=0$, using a comparison theorem for parabolic equations. An attempt was also made to construct an approximate solution, when $\delta<\delta_{\mathrm{cr}}$ and $\theta$ remains small, based on the asymptotic consideration $\alpha \gg 1$. A number of studies on the critical value $\delta_{\mathrm{cr}}$, and its dependence on $\alpha$, have been done by Fenaughty, Lacey and Wake [3], and Kordylewski [5], among others. These studies are based on the time-independent equation corresponding to (1-1), and the dependence of the number of solutions on $\alpha$ was examined.

The construction of upper and lower solutions for (1-1), (1-2), (1-3), and the influence of the initial and boundary data, was studied in a number of papers by Tam, [8], [9], [10], Tam and Kiang [14], Tam and Chapman [13]. These are also for simple geometric configurations. Comparison theorems as well as iteration schemes based on the integral equation derived from (1-1) are used.

In this paper, we present a procedure for the construction of upper and lower solutions. The only restriction on the domain $D$ is that the eigenvalue problem $\nabla^{2} \varphi=-\lambda^{2} \varphi ; \mathbf{x} \in D ; \varphi=0$ on $\partial D$ shall have a discrete spectrum of eigenvalues. 
In II, we reconsider the construction of upper and lower solutions with homogeneous data. The importance of the first eigenfunction is emphasized. In III, we consider the influence of the initial data, with a view of constructing approximate, as well as upper and lower solutions. A two-step linearization of the given problem is introduced. In IV, we give a similar treatment for the influence of the boundary conditions. In V, we consider the effect of the Biot number on the critical parameter. Whenever possible, we compare the present results with those obtained previously. There is good agreement in all cases.

Throughout this paper, we deal with a number of differential or transcendental equations whose solutions depend on the parameters $\alpha$ and $\delta$. They all share the following feature: When $\alpha$ is sufficiently large and $\delta$ is greater than a critical value, say $\delta_{\text {cr }}$, which is particular to the specific equation under consideration, the equation has a unique solution whose maximum value is of order $O\left(e^{\alpha}\right)$. When $\delta$ is less than $\delta_{\mathrm{cr}}$, but greater than an extinction value, say $\delta_{e}$, the equation has three solutions, the largest of order $O\left(e^{\alpha}\right)$ and the smallest of order $O(1)$. When $\delta$ is less than $\delta_{e}$, there is again a unique solution of order $O(1)$. We refer to the large solution as "super-critical" and the small solution as "sub-critical". These terms and the symbols $\delta_{\mathrm{cr}}$ and $\delta_{e}$ are used generically. When we wish to refer to $\delta_{\mathrm{cr}}$ or $\delta_{e}$ of a particular equation, we make them specific by referring to the equation either by number or by context.

\section{Upper and lower solutions with homogeneous data}

In two previous papers [8], [10], the steady state upper solutions for the three simple geometries, viz, slab, circular cylinder, and sphere, were obtained in the manner to be described. Here, the result is presented for a general geometry, and the steady state is that corresponding to homogeneous boundary data. The construction for the lower solutions in [8], [10] was also based on the individual cases. Here, it is for a general geometry and given in terms of the first eigenfunction. The significance of the first eigenfunction was recognized in [9], but has not been exploited in the construction process.

\section{IIa. The steady state}

To start with, we take $\partial / \partial t=h(\mathbf{x})=g(\mathbf{x}, t)=0$, and we focus our attention on the influence of $\delta$. Let $\lambda_{1}^{2}$ be the smallest eigenvalue with corresponding normalized eigenfunctions $\varphi_{1}(x)>0$ of the problem

$$
\begin{gathered}
\nabla^{2} \varphi=-\lambda^{2} \varphi, \quad \mathbf{x} \in D, \\
\varphi(\mathbf{x})=0, \quad \mathbf{x} \text { on } \partial D .
\end{gathered}
$$


Let $k$ be a numerical constant whose magnitude is to be determined. We have:

LEMMA 1. Let $\lambda_{1}^{2}$ and $\varphi_{1}$ be as defined in (2-1), (2-2) with $\varphi_{1 m}$ denoting the maximum value of $\varphi_{1}$ on $D$; and let $k$ be the solution (the smallest if there is more than one solution) of

$$
\lambda_{1}^{2} k \varphi_{1 m}=\delta \exp \left[\alpha k \varphi_{1 m} /\left(\alpha+k \varphi_{1 m}\right)\right] .
$$

Then $u=k \varphi_{1}$ is a lower solution of

$$
\begin{gathered}
\nabla^{2} \theta+\delta f(\theta)=0, \quad \mathbf{x} \in D, \\
\theta(\mathbf{x})=0, \quad \mathbf{x} \in \partial D
\end{gathered}
$$

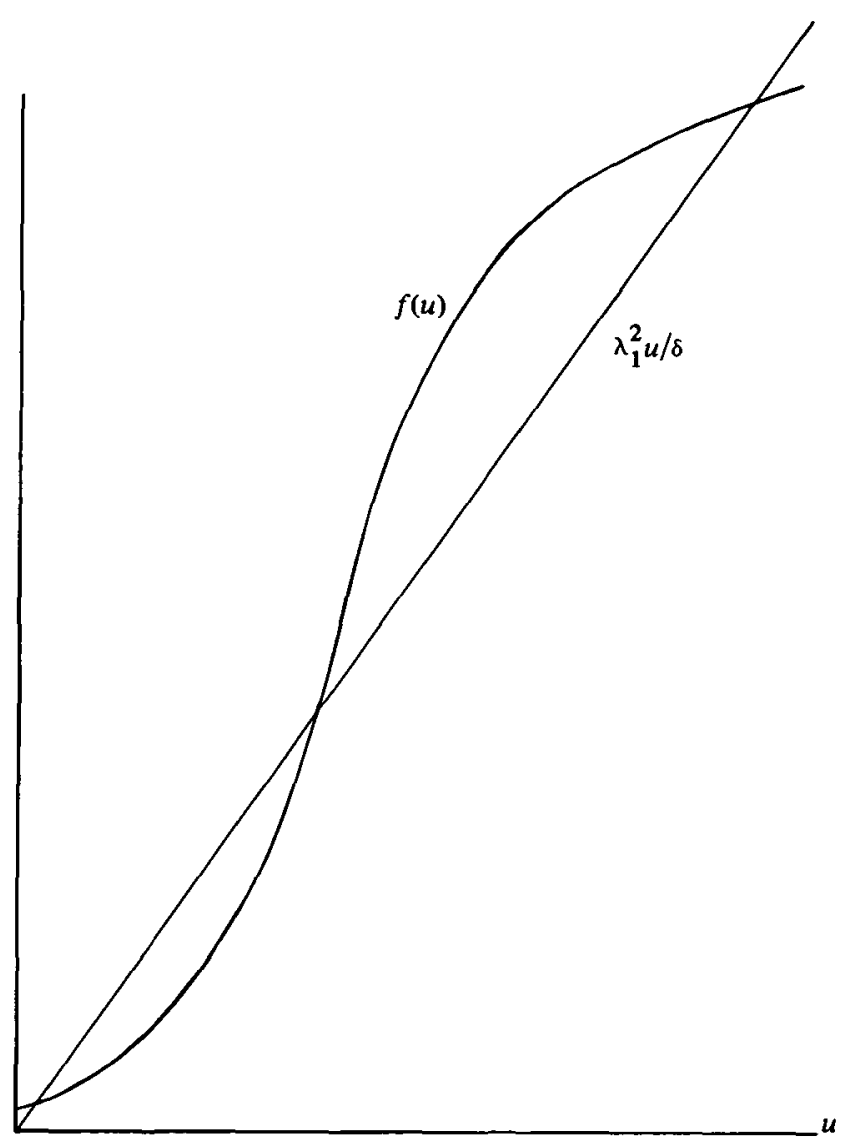

FIGURE I. $\lambda_{1}^{2} u / \delta$ and $f(u)$ vs. $u$. 
Proof. We write $P \theta \equiv \partial \theta / \partial t-\nabla^{2} \theta-\delta f(\theta)$. Hence we have $P u=\lambda_{1}^{2} u-$ $\delta f(u)$. Plotting $\lambda_{1}^{2} u / \delta$ and $f(u)$ versus $u$ in Figure I, it is clear that for $u \leqslant k \varphi_{1 m}$, we have $f(u) \geqslant \lambda_{1}^{2} u / \delta$, with the equality sign holding for $u=k \varphi_{1 m}$. Since $0<\varphi_{1} \leqslant \varphi_{1 m}$, we have $P\left(k \varphi_{1}\right) \leqslant 0$, implying that $k \varphi_{1}$ is a lower solution. Q.E.D.

LEMMA 2. Let $\chi$ be the solution of

$$
\begin{aligned}
& \nabla^{2} \chi=-1, \quad \mathbf{x} \in D, \\
& \chi(\mathbf{x})=0, \quad \mathbf{x} \text { on } \partial D,
\end{aligned}
$$

and $\chi_{m}$ denote the maximum of $\chi$ in $D$. Let $K$ be the solution (the smallest if there is more than one solution) of

$$
K=\delta f\left(K \chi_{m}\right) .
$$

Then $U=K \chi$ is an upper solution of (2-3), (2-4).

Proof. Since $f(\theta)$ is an increasing function of $\theta$, we have

$$
P U=K-\delta f(K \chi) \geqslant K-\delta f\left(K \chi_{m}\right) .
$$

Thus if $K$ is determined from (2-5), we have $P U \geqslant 0$, implying that $U$ is an upper solution. Q.E.D.

Now, from Lemma 1, we can obtain an upper bound for $\delta_{\mathrm{cr}}$, for equations $(2-3),(2-4)$. It is clear from Figure $I$ that if $\delta$ is increased from zero until $\lambda_{1}^{2} u \delta^{-1}-f(u)$ has a double zero at $k \varphi_{1 m}$, a critical value $\bar{\delta}_{\text {cr }}$ is reached. Further increase in $\delta$ will lead to a value of $k$ which is exponentially large, that is, the lower solution for (2-3) and (2-4) becomes super-critical. Thus, a necessary condition for the solution of (2-3) and (2-4) to remain subcritical is that its lower solution $k \varphi_{1}$ must not be exponentially large, implying that we must have $\delta_{\mathrm{cr}}<\bar{\delta}_{\mathrm{cr}}$. The computation of $\bar{\delta}_{\mathrm{cr}}$ is straightforward. We have

$$
\bar{\delta}_{\mathrm{cr}}=\lambda_{1}^{2} u^{*} \exp \left[-\alpha u^{*} /\left(\alpha+u^{*}\right)\right]
$$

where

$$
u^{*}=\frac{1}{2}\left\{\alpha(\alpha-2)-\sqrt{\alpha^{3}(\alpha-4)}\right\} .
$$

In a similar manner, we can also obtain from the upper solution a lower bound for $\delta_{\text {cr }}$, say $\tilde{\delta}_{\text {cr }}$. We have

$$
\tilde{\delta}_{\mathrm{cr}}=K_{1} \exp \left[-\alpha \chi_{m} K_{1} /\left(\alpha+\chi_{m} K_{1}\right)\right]
$$

where

$$
K_{1}=u^{*} / \chi_{m}
$$


Thus, we have $\tilde{\delta}_{\mathrm{cr}}=\bar{\delta}_{\mathrm{cr}} /\left(\chi_{\mathrm{m}} \lambda_{1}^{2}\right)$. We observe that these bounds cease to have any meaning if $\alpha$ is decreased until (2-6) has only one solution regardless of the value of $\boldsymbol{\delta}$.

\section{IIb. The time-dependent case}

With homogeneous initial and boundary data, the time-dependent case can be dealt with in an entirely analogous manner.

LEMMA 1'. Let $\varphi_{1}$ be defined as before and $k(t)$ be a suitably chosen (as detailed in proof ) increasing function of $t$, tending to $k_{m}$ as $t \rightarrow \infty$. Then $u=k \varphi_{1}$ is a lower solution of (1-1), (1-2), (1-3), with $h(\mathbf{x})=g(\mathbf{x}, t)=0$.

PRoof. Let $\varphi_{1 m}$ be the maximum value of $\varphi_{1}(x)$. We first consider $\delta \leqslant \bar{\delta}_{\mathrm{cr}}$. We have

$$
P u=k^{\prime} \varphi_{1}+\lambda_{1}^{2} k \varphi_{1}-\delta f\left(k \varphi_{1}\right) .
$$

If $k(t)$ is determined from

$$
k^{\prime}(t)=\left\{\delta f\left(k \varphi_{1 m}\right)-\lambda_{1}^{2} k \varphi_{1 m}\right\} / \varphi_{1 m}
$$

satisfying $k(0)=0$, it is easily seen that $k(t)$ is a monotonic increasing function, reaching the equilibrium value $k_{m}$ given by

$$
\delta f\left(k_{m} \varphi_{1 m}\right)=\lambda_{1}^{2} k_{m} \varphi_{1 m} .
$$

Thus, we have

$$
P u=\frac{\varphi_{1}}{\varphi_{1 m}}\left\{\delta f\left(k \varphi_{1 m}\right)-\lambda_{1}^{2} k \varphi_{1 m}\right\}-\left\{\delta f\left(k \varphi_{1}\right)-\lambda_{1}^{2} k \varphi_{1}\right\} .
$$

For $0<k<k_{m}$, quantities in both brackets are positive. Thus, we have

$$
P u \leqslant\left(\delta f\left(k \varphi_{1 m}\right)-\lambda_{1}^{2} k \varphi_{1 m}\right)-\left(\delta f\left(k \varphi_{1}\right)-\lambda_{1}^{2} k \varphi_{1}\right) .
$$

From Figure I, it is clear that $\lambda_{1} k \varphi_{1}-\delta f\left(k \varphi_{1}\right)<\lambda_{1} k \varphi_{1 m}-\delta f\left(k \varphi_{1 m}\right)$, so that $P u \leqslant 0$, implying that $u=k \varphi_{1}$ is a lower solution. For $\delta>\bar{\delta}_{\mathrm{cr}}$, the inequality $(2-8)$ no longer holds. In this case, we determined $k(t)$ from the equation

$$
k^{\prime}(t)=\frac{\varepsilon}{\varphi_{1 m}}\left\{\delta f\left(k \varphi_{1 m}\right)-\lambda_{1}^{2} k \varphi_{1 m}\right\}
$$

where $\varepsilon<0$, and so

$$
P u=\varepsilon\left\{\delta f\left(k \varphi_{1 m}\right)-\lambda_{1}^{2} k \varphi_{1 m}\right\}+\left\{\lambda_{1}^{2} k \varphi_{1}-\delta f\left(k \varphi_{1}\right)\right\} .
$$

We observe that the quantity in the second bracket on the right is negative for $k \varphi_{1}<k_{m} \varphi_{1 m}$. Thus, if $\varepsilon$ is sufficiently small, the right side of (2-9) remains negative for $k \varphi_{1 m}<k_{m} \varphi_{1 m}$, and so we have $P u \leqslant 0$, again implying that $u=k \varphi$ is a lower solution. In this case, the solution of $(2-7)$ is $O\left(e^{\alpha}\right)$. Q.E.D. 
As for the upper solution, we observe that for $g(x, t)=h(\mathbf{x})=0$, the upper solution for the time-independent case also serves an upper solution for the time-dependent case. If we want to have an explicit time-dependent upper solution, we can use the upper solution for the time-dependent case to linearize the given differential equation. The procedure is straightforward, and will not be pursued.

\section{The influence of the initial data}

For $\delta$ in a certain range, and for $\alpha$ sufficiently large, the steady state equation admits more than one solution. The intial and/or boundary data then determine which steady state is realized. The influence of the initial data was investigated in [9], [10]. The approach there was to re-write the partial differential equation as an integral equation and to use an iteration scheme based on an asymptotic analysis of the large time behaviour of the solution. The initial data were used in the first iterate.

In the present paper, we approach the problem from the standpoint of constructing upper and lower solutions. We begin by considering an associated ordinary differential equation which we believe to contain the essence of the given non-linear problem. We then show that upper and lower solutions to the given problem can be constructed by using suitable multiples of the solution of the ordinary differential equation to linearize the given problem. A similar idea has been used by the author in the study of swirling-flow boundary layers in fluid mechanics [12]. Lastly, we compare the current result with those in [9], [10].

IIIa. The associated ordinary differential equation

Let $H(\mathbf{x}, t)$ denote the solution of the IBVP

$$
\begin{gathered}
H_{t}-\nabla^{2} H=0, \\
H(\mathbf{x}, 0)=h(\mathbf{x}) \geqslant 0, \quad H(\mathbf{x}, t)=0, \quad \mathbf{x} \in \partial D .
\end{gathered}
$$

We have

$$
H(\mathbf{x}, t)=G(\mathbf{x}, \xi, t) \cdot h(\mathbf{x}) \equiv \int_{D} G(\mathbf{x}, \boldsymbol{\xi}, t) h(\xi) d V
$$

where

$$
G(\mathbf{x}, \xi, t)=\sum_{n=1}^{\infty} e^{-\lambda_{n}^{2} t} \varphi_{n}(\mathbf{x}) \varphi_{n}(\xi)
$$


and $\left\{\lambda_{n}^{2}\right\}$ and $\left\{\varphi_{n}(\mathbf{x})\right\}$ are respectively the eigenvalues and normalized eigenfunctions of $(2-1)$ and (2-2). Let $T(t)$ denote the solution of the IVP

$$
\begin{gathered}
\frac{d T(t)}{d t}=-\lambda_{1}^{2} T(t)+\delta \int_{D} \varphi_{1}(\xi) \exp \left[\frac{\alpha\left(T(t) \varphi_{1}(\xi)+H(\xi, t)\right)}{\alpha+T(t) \varphi \varphi_{1}(\xi)+H(\xi, t)}\right] d V, \\
T(0)=0 .
\end{gathered}
$$

Equivalently, $T$ can be expressed as the solution of the integral equation

$$
T=\delta e^{-\lambda_{1}^{2} t} \int_{0}^{t} e^{\lambda_{1}^{2} \tau} \varphi_{1}(\xi) \cdot \exp \left[\frac{\alpha\left(T(\tau) \varphi_{1}(\xi)+H(\xi, \tau)\right)}{\alpha+T(\tau) \varphi_{1}(\xi)+H(\xi, \tau)}\right] d \tau .
$$

We observe that, since all eigenfunction components of $H(\mathbf{x}, t)$ decrease exponentially to zero, the equilibrium values of $T$ are the solutions of the equation

$$
\frac{\lambda_{1}^{2} T}{\delta}=\int_{D} \varphi_{1}(\xi) \exp \left[\frac{\alpha T \varphi_{1}(\xi)}{\alpha+T \varphi_{1}(\xi)}\right] d V .
$$

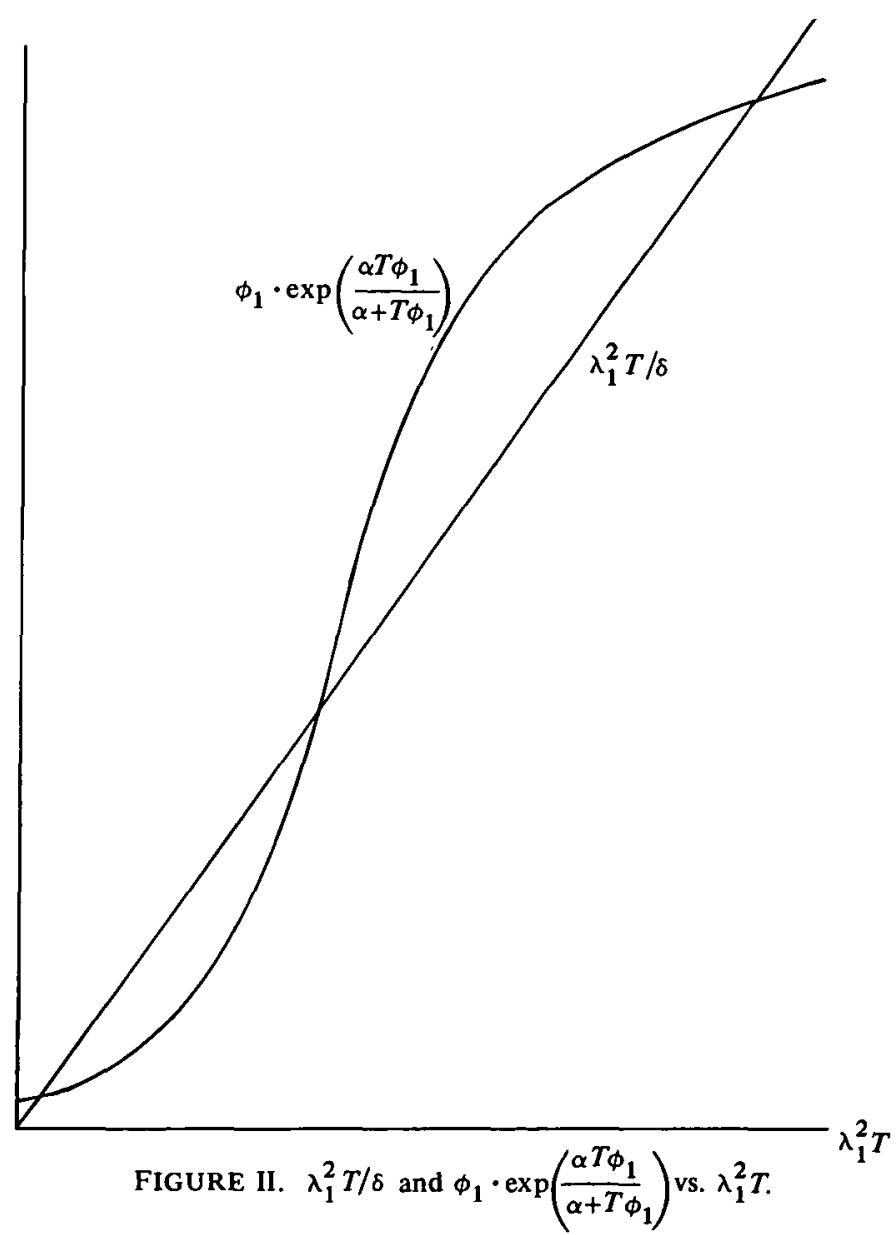


Plotting the two terms of (3-6) as solid lines in Figure II, we see that there are three solutions in the situation depicted. We label the minimum solution as sub-critical, and the maximum as super-critical. Clearly, there are two threshold values for $\delta$, viz, $\delta_{e}$, and $\delta_{\mathrm{cr}}$, such that for $\delta>\delta_{\mathrm{cr}}$, there is only one (super-critical) solution; and for $\delta<\delta_{e}$, there is again only one (sub-critical) solution. We reiterate that at this point, the terms super-critical and sub-critical, as well as the symbols $\delta_{e}$ and $\delta_{\mathrm{cr}}$, refer to (3-5) only. Suppose $\delta_{e}<\delta<\delta_{\mathrm{cr}}$. We first consider the particular case $h(\mathbf{x})=c_{1} \varphi_{1}(\mathbf{x})$, where $c_{1}$ is a constant. We have $H(\mathbf{x}, t)=$ $c_{1} \exp \left(-\lambda_{1}^{2} t\right) \varphi_{1}(\mathbf{x})$. Let $y=T(t)+c_{1} \exp \left(-\lambda_{1}^{2} t\right)$. In terms of $y,(3-3)$ and (3-4) become

$$
\begin{gathered}
\frac{d y}{d t}=-\lambda_{1}^{2} y+\delta \int_{D} \varphi_{1}(\xi) \exp \left\{\frac{\alpha y(t) \varphi_{1}(\xi)}{\alpha+y(t) \varphi_{1}(\xi)}\right\} d V, \\
y(0)=c_{1} .
\end{gathered}
$$

Except for the changed initial data, $y(t)$ has the same equilibrium values as $T(t)$. Thus, it is clear from Figure II that if $y(0)=c_{1}>v^{*} / \lambda_{1}^{2}$, where $v^{*}$ is the middle intersection of the curve $\varphi_{1} \exp \left[\alpha T \varphi_{1} /\left(\alpha+T \varphi_{1}\right)\right]$ with the straight line $\lambda_{1}^{2} T / \delta$, then $y$ reaches the super-critical state, and if $y(0)<v^{*} / \lambda_{1}^{2}, y$ reaches the sub-critical state. We note that $y(0) \gtrless v^{*} / \lambda_{1}^{2}$ implies that

$$
\delta \int_{D} \varphi_{1}(\xi) \exp \left\{\frac{\alpha h(\xi)}{\alpha+h(\xi)}\right\} d V \gtrless v^{*}
$$

In terms of $T$, we have

$$
\frac{d T(0)}{d t}=\delta \int_{D} \varphi_{1}(\xi) \exp \left\{\frac{\alpha h(\xi)}{\alpha+h(\xi)}\right\} d V .
$$

Thus, we conclude that $T$ reaches the super-critical state if $d T(0) / d t>v^{*}$, and it reaches the sub-critical state if $d T(0) / d t<v^{*}$.

We now consider the case of an arbitrary $h(\mathbf{x})$. We write

$$
H(\mathbf{x}, t)=\sum_{n=1}^{\infty} c_{n} \exp \left(-\lambda_{n}^{2} t\right) \varphi_{n}(\mathbf{x}) \equiv c_{1} \exp \left(-\lambda_{1}^{2} t\right) \varphi_{1}(\mathbf{x})+J(\mathbf{x}, t)
$$

where

$$
c_{n}=\int_{D} \varphi_{n}(\mathbf{x}) h(\mathbf{x}) d V .
$$

We again let $y=T+c_{1} e^{-\lambda_{1}^{2} t}$. Equations (3-3) and (3-4) become

$$
\begin{gathered}
\frac{d y}{d t}=-\lambda_{1}^{2} y+\delta \varphi_{1} \cdot \exp \left\{\frac{\alpha\left[y \varphi_{1}+J\right]}{\alpha+y \varphi_{1}+J}\right\}, \\
y(0)=c_{1} ; \quad \frac{d y(0)}{d t}=-\lambda_{1}^{2} c_{1}+\delta \varphi_{1} \cdot e^{\alpha h /(\alpha+h)} .
\end{gathered}
$$


We seek conditions under which $y$ tends to the super-critical state. Let $I(t)$ be defined as

$$
I(t)=\varphi_{1}(\mathbf{x}) \cdot \exp \left\{\frac{\alpha\left(c_{1} \varphi_{1}(\mathbf{x})\right)+J(x, t)}{\alpha+c_{1} \varphi_{1}(x)+J(x, t)}\right\} .
$$

Let $h(\mathbf{x})$ be such that the following two conditions are satisfied.

$$
I(0)=\varphi_{1} \cdot \exp \left\{\frac{\alpha h}{\alpha+h}\right\}>\varphi_{1} \cdot \exp \left\{\frac{\alpha c_{1} \varphi_{1}}{\alpha+c_{1} \varphi_{1}}\right\}
$$

where $c_{1}>v^{*} / \lambda_{1}^{2}$

$$
\frac{d I(t)}{d t}<0 \text { for } 0<t<\infty .
$$

Since $\delta \varphi_{1} \cdot \exp \left\{\alpha c_{1} \varphi_{1} /\left(\alpha+c_{1} \varphi_{1}\right)\right\}>-\lambda_{1}^{2} c_{1}$, we have $d y(0) / d t>0$.

Suppose $y$ does not tend to the super-critical state. Then $d y / d t$ must become negative and there exists $t_{1}<\infty$ at which $y\left(t_{1}\right)=c_{1}$, with

$$
\frac{d y\left(t_{1}\right)}{d t}=-\lambda_{1}^{2} c_{1}+\delta \varphi_{1} \cdot \exp \left\{\frac{\alpha\left(c_{1} \varphi_{1}+J\left(x, t_{1}\right)\right)}{\alpha+c_{1} \varphi_{1}+J\left(x, t_{1}\right)}\right\} \leqslant 0 .
$$

However, by (3-12), $I(t)$ decreases monotonically to $\varphi_{1} \exp \left[\alpha c_{1} \varphi_{1} /\left(\alpha+c_{1} \varphi_{1}\right)\right]$. Thus, we have

$$
\begin{aligned}
\delta \varphi_{1} \cdot \exp \left\{\frac{\alpha\left(c_{1} \varphi_{1}+J\left(x, t_{1}\right)\right)}{\alpha+c_{1} \varphi_{1}+J\left(x, t_{1}\right)}\right\}-\lambda_{1}^{2} c_{1} & >\delta \varphi_{1} \cdot \exp \left\{\frac{\alpha c_{1} \varphi_{1}}{\alpha+c_{1} \varphi_{1}}\right\}-\lambda_{1}^{2} c_{1} \\
& >0,
\end{aligned}
$$

contradicting $d y\left(t_{1}\right) / d t \leqslant 0$. Thus, $y$ cannot resume to value $c_{1}$, and so $y$ tends to the super-critical state as $t \rightarrow \infty$. We summarize this result in

Lemma 3. Let $v^{*}$ and $I(t)$ be as defined. If $h(\mathbf{x})$ satisfies the two conditions (3.12a) and (3.12b), then the solution of equations (3-10), (3-11), and hence $T$, tends to the super-critical value.

In an analogous manner, we have

Lemma $3^{\prime}$. Let $v^{*}$ and $I(t)$ be as defined in Lemma 3. If $h(\mathbf{x})$ satisfies the two conditions

(1) $I(0)=\varphi_{1} \cdot \exp \{\alpha h / \alpha+h\}<\varphi_{1} \cdot \exp \left\{\alpha c_{1} \varphi_{1} /\left(\alpha+c_{1} \varphi_{1}\right)\right\}$, where $c_{1}<$ $v^{*} / \lambda_{1}^{2}$

(2) $d T / d t<0$ for $0<t<\infty$, then the solution of equations (3-10), (3-11), and hence $T$, tends to the subcritical value. 
A different set of sufficient conditions giving the same conclusion as in the above Lemmas can be formulated. We observe that $J(x, t)$ is the solution of the heat equation

$$
\frac{\partial J}{\partial t}=\nabla^{2} J ; \quad \mathbf{x} \in D ; t>0
$$

subject to

$$
J(\mathbf{x}, 0)=\sum_{n=2}^{\infty} c_{n} \varphi_{n}(\mathbf{x}) ; \quad J=0 \quad \text { on } \partial D .
$$

Suppose $-a \varphi_{1}(\mathbf{x})<J(\mathbf{x}, 0)<A \varphi_{1}(\mathbf{x})$ for suitably determined non-negative $a, A$. It is readily seen that

$$
-a \exp \left[-\lambda_{1}^{2} t\right] \varphi_{1}(\mathbf{x})<J(\mathbf{x}, t)<A \exp \left[-\lambda_{1}^{2} t\right] \varphi_{1}(\mathbf{x}) .
$$

Now we consider the equation

$$
\frac{d \tilde{y}}{d t}=-\lambda_{1}^{2} \tilde{y}+\delta \int_{D} \varphi_{1}(\xi) \exp \left[\frac{\alpha\left(\tilde{y}(t) \varphi_{1}(\mathbf{x})-a \exp \left(-\lambda_{1}^{2} t\right) \varphi_{1}(\mathbf{x})\right)}{\alpha+\tilde{y}(t) \varphi_{1}(\mathbf{x})-a \exp \left(-\lambda_{1}^{2} t\right) \varphi_{1}(\mathbf{x})}\right] d V
$$

with

$$
\tilde{y}(0)=c_{1}, \quad \frac{d \tilde{y}(0)}{d t}=-\lambda_{1}^{2} c_{1}+\delta \varphi_{1} \cdot \exp \left\{\frac{\alpha\left(c_{1}-a\right) \varphi_{1}}{\alpha+\left(c_{1}-a\right) \varphi_{1}}\right\} .
$$

Recalling that the function $\exp [\alpha u /(\alpha+u)]$ is an increasing function of $u$, it follows from a comparison theorem for ordinary differential equation that $\tilde{y}<y$. We note that the value $v^{*}$ determined before is also the middle intersection of the curve $\varphi_{1} \exp \left[\alpha \tilde{y} \varphi_{1} /\left(\alpha+\tilde{y} \varphi_{1}\right)\right]$ with $\lambda_{1}^{2} \tilde{y} / \delta$. Thus, if we require the function $h(x)$ to satisfy the two conditions (1) $c_{1} \geqslant v^{*} / \lambda_{1}^{2}$ and

$$
\delta \varphi_{1} \cdot \exp \left\{\alpha\left(c_{1}-a\right) \varphi_{1} /\left(\alpha+\left(c_{1}-a\right) \varphi_{1}\right)\right\}-\lambda_{1}^{2} c_{1}>0
$$

we have $d \tilde{y}(0) / d t>0$. We claim $d \tilde{y} / d t$ can tend to zero only as $t \rightarrow \infty$. Suppose the contrary is true. Let $d \tilde{y} / d t=0$ at $t_{1}$, with $d \tilde{y} / d t>0$ for $0 \leqslant t<t_{1}$. Then at $t_{1}$, we have

$$
\begin{aligned}
\frac{d^{2} \tilde{y}}{d t^{2}}= & \delta \alpha^{2} \int_{D} \frac{a \lambda_{1}^{2} \exp \left(-\lambda_{1}^{2} t\right) \varphi_{1}^{2}(\mathbf{x})}{\left[\alpha+\tilde{y}(t) \varphi_{1}(\mathbf{x})-a \exp \left(-\lambda_{1}^{2} t\right) \varphi_{1}(\mathbf{x})\right]^{2}} \\
& \cdot \exp \left[\frac{\alpha\left(\tilde{y}(t) \varphi_{1}(\mathbf{x})-a \exp \left(-\lambda_{1}^{2} t\right) \varphi_{1}(\mathbf{x})\right)}{\alpha+\tilde{y}(t) \varphi_{1}(\mathbf{x})-a \exp \left(-\lambda_{1}^{2} t\right) \varphi_{1}(\mathbf{x})}\right] d V
\end{aligned}
$$

which is positive, leading to a contradiction. Thus $d \tilde{y} / d t>0$ for all finite $t$, and $\tilde{y}$, and hence $y$ and $T$ all tend to the same super-critical value. The two conditions imposed on $h(\mathbf{x})$ together imply

$$
\delta \int_{D} \varphi_{1}(\xi) \exp \left\{\frac{\alpha\left[h(\mathbf{x})-J(\mathbf{x}, 0)-a \varphi_{1}(\mathbf{x})\right]}{\alpha+h(\mathbf{x})-J(\mathbf{x}, 0)-a \varphi_{1}(\mathbf{x})}\right\} d V>\lambda_{1}^{2} c_{1}>v^{*} .
$$


We can summarize the above result as:

LEMMA 4. Let $v^{*}, J$ and $a$ be as defined above. If $h(\mathbf{x})$ satisfies (3-13), the solution of (3-10), (3-11), and hence $T$, tends to the super-critical value.

In an analogous manner, we have:

LEMmA $4^{\prime}$. Let $v^{*}, J$ and $A$ be as defined above. If $h(\mathbf{x})$ satisfies the condition

$$
\delta \int_{D} \varphi_{1}(\xi) \exp \left\{\frac{\alpha\left[h(\mathbf{x})-J(\mathbf{x}, 0)+A \varphi_{1}(\mathbf{x})\right]}{\alpha+h(\mathbf{x})-J(\mathbf{x}, 0)+A \varphi_{1}(\mathbf{x})}\right\} d V<\lambda_{1}^{2} c_{1}<v^{*}
$$

the solution of (3-10), (3-11), and hence $T$, tends to the sub-critical value.

We observe that the quantity $\delta \varphi_{1} \exp [\alpha h /(\alpha+h)]$ is bounded from below by the integral in (3-13), and from above by the integral in (3-14). These bounds are close when the derivations $\left(J(\mathbf{x}, 0)+a \varphi_{1}\right)$ and $\left(A \varphi_{1}-j(\mathbf{x}, 0)\right)$ are small.

We have considered in some detail the behaviour of a function $T(t)$. In the next section, we shall use this function in the construction of upper and lower solutions for $\theta(\mathbf{x}, t)$; and also obtain an approximate solution for $\theta(\mathbf{x}, t)$.

\section{IIIb. Construction of upper and lower solutions}

Let $H$ and $T$ be as defined in (3-1) and (3-5). Let $m(\mathbf{x})>0$ be a function to be determined. We consider the IBVP

$$
\chi_{t}-\nabla^{2} \chi=\delta \exp \left[\frac{\alpha\left(m T \varphi_{1}+H\right)}{\alpha+m T \varphi_{1}+H}\right]
$$

subject to $\chi(\mathbf{x}, 0)=h(\mathbf{x}) ; \chi(\mathbf{x}, t)=0, x \in \partial D$. Since the equation is linear, its solution can be obtained readily. We have (see [2], page 291)

$$
\chi(\mathbf{x}, t)=H(\mathbf{x}, t)+\delta \int_{0}^{t} G(\mathbf{x}, \xi, t \tau) \cdot \exp \left[\frac{\alpha\left(m T(\tau) \varphi_{1}(\xi)+H(\xi, \tau)\right)}{\alpha+m T(\tau) \varphi_{1}(\xi)+H(\xi, \tau)}\right] d \tau
$$

Our objective is to choose $m(\mathbf{x})$ so that $\chi(\mathbf{x}, t)$ is a lower solution of equation $(1-1),(1-2)$ and (1-3), with $g(x, t)=0$, i.e. we wish to make

$$
P \chi=\chi_{t}-\nabla^{2} \chi-\delta \exp \left(\frac{\alpha \chi}{\alpha+\chi}\right) \leqslant 0 \text {. }
$$

Now, we have

$$
P \chi=\delta\left\{\exp \left[\frac{\alpha\left(m T(t) \varphi_{1}(\mathbf{x})+H(\mathbf{x}, t)\right)}{\alpha+m T(t) \varphi_{1}(\mathbf{x})+H(\mathbf{x}, t)}\right]-\exp \left[\frac{\alpha \chi(\mathbf{x}, t)}{\alpha+\chi(\mathbf{x}, t)}\right]\right\}
$$


Since the function $\exp [\alpha u /(\alpha+u)]$ is an increasing function of $u$, we will have $P \chi \leqslant 0$ if we can choose $m$ to make

$$
m T \varphi_{1}+H \leqslant H+\delta \int_{0}^{t} G(\mathrm{x}, \xi, t-\tau) \cdot \exp \left[\frac{\alpha\left(m T(\tau) \varphi_{1}(\xi)+H(\xi, \tau)\right)}{\alpha+m T(\tau) \varphi_{1}(\xi)+H(\xi, \tau)}\right] d \tau
$$

or

$$
m T(t) \varphi_{1}(\mathbf{x}) \leqslant \delta \int_{0}^{t} G(\mathbf{x}, \xi, t-\tau) \cdot \exp \left\{\frac{\alpha\left(m T(\tau) \varphi_{1}(\xi)+H(\xi, \tau)\right)}{\alpha+m T(\tau) \varphi_{1}(\xi)+H(\xi, \tau)}\right\} d \tau .
$$

From equation (3-5), we have

$$
\begin{aligned}
m T(t) \varphi_{1}(\mathbf{x}) & =\delta \int_{0}^{t} G(\mathbf{x}, \boldsymbol{\xi}, t-\tau) \cdot \exp \left[\frac{\alpha\left(m T(\tau) \varphi_{1}(\xi)+H(\xi, \tau)\right)}{\alpha+m T(\tau) \varphi_{1}(\xi)+H(\xi, \tau)}\right] d \tau \\
+ & \delta m \int_{0}^{t} e^{-\lambda_{1}^{2}(t-\tau)} \varphi_{1}(\mathbf{x}) \varphi_{1}(\xi) \cdot \exp \left[\frac{\alpha\left(T(\tau) \varphi_{1}(\xi)+H(\xi, \tau)\right)}{\alpha+T(\tau) \varphi_{1}(\xi)+H(\xi, \tau)}\right] d \tau \\
& -\delta \int_{0}^{t} G(\mathbf{x}, \boldsymbol{\xi}, t-\tau) \cdot \exp \left[\frac{\alpha\left(m T(\tau) \varphi_{1}(\xi)+H(\xi, \tau)\right)}{\alpha+m T(\tau) \varphi_{1}(\xi)+H(\xi, \tau)}\right] d \tau .
\end{aligned}
$$

Since the exponential function in the integrands is bounded between 1 and $\exp (\alpha)$, and both $\varphi_{1}$ and $G$ are positive, it is clear that by choosing $m$ sufficiently small, the sum of the second and third integrals on the right of the above equation can be made to be negative, thus ensuring that equation (3-16) is satisfied, and therefore implying that the function $\chi(x, t)$ given by (3-15) is a lower solution. An entirely analogous procedure with $M>0$ replacing $m$ yields an upper solution. By examining equation (3-14) we see that the deviation of $m$ and $M$ from unity is governed by the magnitude of

$$
\left\{\sum_{n=2}^{\infty} e^{-\lambda_{n}^{2}(t-\tau)} \varphi_{n}(\mathbf{x}) \varphi_{n}(\xi)\right\} \cdot \exp \left\{\frac{\alpha\left(T(\tau) \varphi_{1}(\xi)+H(\xi, \tau)\right)}{\alpha+T(\tau) \varphi_{1}(\xi)+H(\xi, \tau)}\right\} .
$$

Since the shape of the exponential function is not too different from that of const. $\varphi_{1}(\xi)$, we expect the above quantity, and hence the deviation of $m$ and $M$ from unity, to be small. In view of this, we can consider $\chi$ as given by (3-15) with $m=1$ as an approximation for $\theta$, the solution of the original problem. An examination of this solution shows that if $T$ becomes large, (labelled as supercritical), $\chi$ will also be large, and if $T$ remains small (labelled as sub-critical), $\chi$ will also remain small. Thus, the dependence of $T$ on $\delta$ and $h(\mathbf{x})$ implies a corresponding dependence of $\chi$ on $\delta$ and $h(x)$, and this is the essence of the two-step linearization that links $T, \chi$ and $\theta$ together.

As an illustration, we have computed $m(x)$ and $M(x)$ in the form of step functions, for the case of an infinite slab, $0<x<1$, taking $\alpha=20$, and 
$H(\xi, \tau)=5 \exp \left(-\lambda_{1}^{2} \tau\right) \varphi_{1}(\xi)=5 \sqrt{2} \exp \left(-\pi^{2} \tau\right) \sin \pi \xi$. We carried out the computation for a time interval of $0<t \leqslant 1.5$, with a step size of 0.1 in $t$ as well as $x$. We find

$$
m(x)= \begin{cases}1.0, & 0<x<0.3, \quad 0.7<x<1, \\ 0.75, & 0.3 \leqslant x \leqslant 0.7,\end{cases}
$$

and

$$
M(x)=\left\{\begin{array}{lll}
3.1, & 0<x<0.2, & 0.8<x<1, \\
1.1, & 0.2 \leqslant x \leqslant 0.8 .
\end{array}\right.
$$

Thus, for $0<t<1.5, \chi(m)$ and $\chi(M)$ are respectively lower and upper solutions of (1-1), (1-2) and (1-3) with $g=0$ and $h=5 \sqrt{2} \sin \pi x$. In this case, to use $m=1$ to provide an approximate solution gives quite reasonable results.

\section{IIIc. Comparison with previous result}

Previously, the author has considered the influence of the initial value by using an iteration scheme, together with certain large-time asymptotic analysis. We now demonstrate that the present approach yields similar results. Retaining the notation in [9], [10], [11] we have

$$
\theta_{n+1} \sim K_{n} \delta U_{1} / \lambda_{1}^{2}
$$

and

$$
\theta_{n+2}-K_{n+1} \delta U_{1} / \lambda_{1}^{2}
$$

where

$$
K_{n+1}=U_{1} \cdot \exp \left\{\frac{\alpha K_{n} \delta U_{1} / \lambda_{1}^{2}}{\alpha+K_{n} \delta U_{1} / \lambda_{1}^{2}}\right\}
$$

Here, $U_{1}$ is the first normalized eigenfunction with corresponding eigenvalue $\lambda_{1}^{2}$. If we rewrite $K_{0} \delta / \lambda_{1}^{2}$ as $T$, i.e. $K_{n} \equiv \lambda_{1}^{2} T / \delta$, then the above equation becomes $K_{n+1}=U_{1} \exp \left[\alpha T U_{1} /\left(\alpha+T U_{1}\right)\right]$. Our previous result shows that if $K_{n+1}>K_{n}$, then the iterates $\left\{\theta_{n}\right\}$ increase to a limit, and if $K_{n+1}<K_{n},\left\{\theta_{n}\right\}$ decrease to a limit. In particular, if $\delta$ is in a range permitting multiple steady-state solutions, then the solution will be super-critical if $K_{n}>v^{*} / \delta$, and sub-critical if $K_{n}<v^{*} / \delta$, where $v^{*}$ is the middle intersection of the curve $K_{n+1}$ with $K_{n}$ plotted against $v=K_{n} \delta$. Referring to equation (3-6) and Figure II, it is clear that the comparison of $K_{n}$ with $K_{n+1}$ is exactly the comparison of the two terms of (3-6). Thus, the values for $\delta_{e}, \delta_{\mathrm{cr}}$ and $v^{*}$ obtained from the previous asymptotic results are precisely those obtainable from the associated ordinary differential equation studied here. The initial data $\theta(\mathbf{x}, 0)=h(\mathbf{x})$ were used previously to calculate $U_{1} \exp [\alpha h /(\alpha+h)]$, which was then compared with $v^{*} / \delta$. The solution $\theta$ reaches the super-critical state if $\delta U_{1} \exp [\alpha h /(\alpha+h)]>v^{*}$, and reaches the sub-critical 
state if $\delta U_{1} \exp [\alpha h /(\alpha \in h)]<v^{*}$. In Lemmas 3.3', 4.4', we have obtained sufficient conditions on $h$ for $T$ to become sub- or super-critical. By inference, these are also sufficient conditions for $\chi$ to be sub- or super-critical. It does appear that the asymptotic result is consistent with the present results. We have thus obtained some relevant information on the initial data and parameter dependence for the original nonlinear parabolic equation by studying an associated ordinary differential equation.

\section{Influence of the boundary condition}

In this section, we examine the influence of the boundary condition in determining the nature of the steady state. Thus, we set the initial value to zero. We first partition the problem into two parts so as to make the effect of the boundary condition more transparent.

Let $\theta=P+\psi$, where $P$ is governed by the system

$$
\begin{gathered}
P(\mathbf{x}, 0)=0 ; \quad P(\mathbf{x}, t)=g(\mathbf{x}, t) \geqslant 0, \mathbf{x} \in \partial D,
\end{gathered}
$$

and $\psi$ is governed by the system

$$
\begin{gathered}
\psi_{t}-\nabla^{2} \psi=\delta f(P+\psi), \\
\psi(\mathbf{x}, 0)=0 ; \quad \psi(\mathbf{x}, t)=0, \mathbf{x} \in \partial D .
\end{gathered}
$$

The solution of (4-1) and (4-2) is given by (see [2], page 290)

$$
P(\mathbf{x}, t)=-\int_{0}^{t} \int_{S} \frac{\partial G(\mathbf{x}, \boldsymbol{\xi}, t-\tau)}{\partial n_{\xi}} g(\xi, \tau) d S_{\xi} d \tau .
$$

We observe that $g \geqslant 0$ implies that $P(\mathbf{x}, t) \geqslant 0$, and we suppose that $g$ is suitably restricted to ensure that $P(\mathbf{x}, \infty)$ exists. Here, we differentiate between the two cases $P(\mathbf{x}, \infty)=0$ and $P(\mathbf{x}, \infty)=C(x) \not \equiv 0$.

To begin with, let $\delta<\delta_{\mathrm{cr}}$, and let

$$
B=\max _{0<t<\infty} \int_{D} \varphi_{1} \exp \left(\frac{\alpha P}{\alpha+P}\right) d V .
$$

Suppose $B$ occurs at $t=t_{1}>0$. Since $t_{1}>0$ implies $\psi\left(x, t_{1}\right)>0$, it is clear that at $t_{1}$, we have

$$
\int_{D} \varphi_{1} \exp \left(\frac{\alpha(P+\psi)}{\alpha+P+\psi}\right) d V>B
$$

With $t_{1}$ taken as the new initial point for (4-3) and (4-4), the same consideration as in section III shows that for $\psi$, and hence $\theta$, to reach the super-critical state, it is sufficient to have $(P+\psi) t_{1}$ satisfying conditions imposed on $h$ as detailed in 
IIIa. However, since we do not know the value of $\psi$ at $t_{1}$, we have to be content with the sufficiency condition being imposed on $\delta B$. For the case of $P(\mathrm{x}, \infty)=0$, the above is the only information we can obtain. However, more can be said about the case of $P(x, \infty)>0, x \in D$; and in what follows, our attention is confined to this case. We note that the problem of a slab with unsymmetric boundary temperatures considered by Tam and Chapman [13], among others, belongs to this case.

IVa. The associated ordinary differential equation

Let $T(t)$ be the solution of the ordinary differential equation

$$
\frac{d T}{d t}=-\lambda_{1}^{2} T+\delta \int_{D} \varphi_{1}(\xi) \exp \left[\frac{\alpha\left(T(t) \varphi_{1}(\xi)+P(\xi, t)\right)}{\alpha+T(t) \varphi_{1}(\xi)+P(\xi, t)}\right] d V
$$

subject to $T(0)=0$. Equivalently, $T$ can be expressed as the solution of the integral equation

$$
T=\delta \int_{0}^{t} e^{-\lambda_{1}^{2}(t-\tau)} \varphi_{1}(\xi) \cdot \exp \left[\frac{\alpha\left(T(\tau) \varphi_{1}(\xi)+P(\xi, \tau)\right)}{\alpha+T(\tau) \varphi_{1}(\xi)+P(\xi, \tau)}\right] d \tau
$$

Here, we have used $T$ to denote a somewhat different quantity as in Section III, but the possibility of confusion is more than compensated for by avoiding a proliferation of symbols. In Figure III, we superpose on Figure II the new curve

$$
\varphi_{1} \cdot \exp \left[\frac{\alpha\left(T \varphi_{1}+P(\xi, \infty)\right)}{\alpha+T \varphi_{1}+P(\xi, \infty)}\right]
$$

It is clear that $P(\xi, \infty)>0$ implies that the new curve always lies above the curve $\varphi_{1} \cdot \exp \left[\alpha T \varphi_{1} /\left(\alpha+T \varphi_{1}\right)\right]$. Further, for a given $\delta<\delta_{\mathrm{cr}}$, it is clear that if $P(\xi, \infty)$ is sufficiently large, the new curve will intersect the straight line $\lambda_{1}^{2} T / \delta$ only at one point. Moreover, since

$$
\varphi_{1} \cdot \exp \left\{\frac{\alpha\left(T \varphi_{1}+P(\xi, \infty)\right)}{\alpha+T \varphi_{1}+P(\xi, \infty)}\right\}
$$

is bounded from above by $\varphi_{1} \cdot e^{\alpha}$ no matter how large $P$ is, it is clear that the intersection point decreases monotonically to zero with $\delta$.

We now pose the same question as in Section III: for a given $\delta, \delta_{e}<\delta<\delta_{\mathrm{cr}}$, such that $\lambda_{1}^{2} T$ intersects

$$
\varphi_{1} \cdot \exp \left\{\frac{\alpha T \varphi_{1}}{\alpha+T \varphi_{1}}\right\}
$$

at three points, how large must $P(x, \infty)$ be for the solution of $(4-7)$ to be super-critical? 
We construct the curve

$$
\varphi_{1} \cdot \exp \left(\frac{\alpha\left(T \varphi_{1}+C\right)}{\alpha+T \varphi_{1}+C}\right)
$$

where $C$ is a constant. We increase the value of $C$ from zero to $C^{*}$, at which

$$
\varphi_{1} \cdot \exp \left(\frac{\alpha\left(T \varphi_{1}+C^{*}\right)}{\alpha+T \varphi_{1}+C^{*}}\right)
$$

becomes tangent to $\lambda_{1}^{2} T / \delta$ at the point $\lambda_{1}^{2} T=b^{*}$, as depicted schematically in Figure III. Now, suppose for a given $g(\mathbf{x}, t)$, we have

$$
\varphi_{1} \cdot \exp \left[\frac{\alpha\left(T \varphi_{1}+P(\xi, \infty)\right)}{\alpha+T \varphi_{1}+P(\xi, \infty)}\right]>\varphi_{1} \cdot \exp \left[\frac{\alpha\left(T \varphi_{1}+C^{*}\right)}{\alpha+T \varphi_{1}+C^{*}}\right] \text { for } T>0,
$$

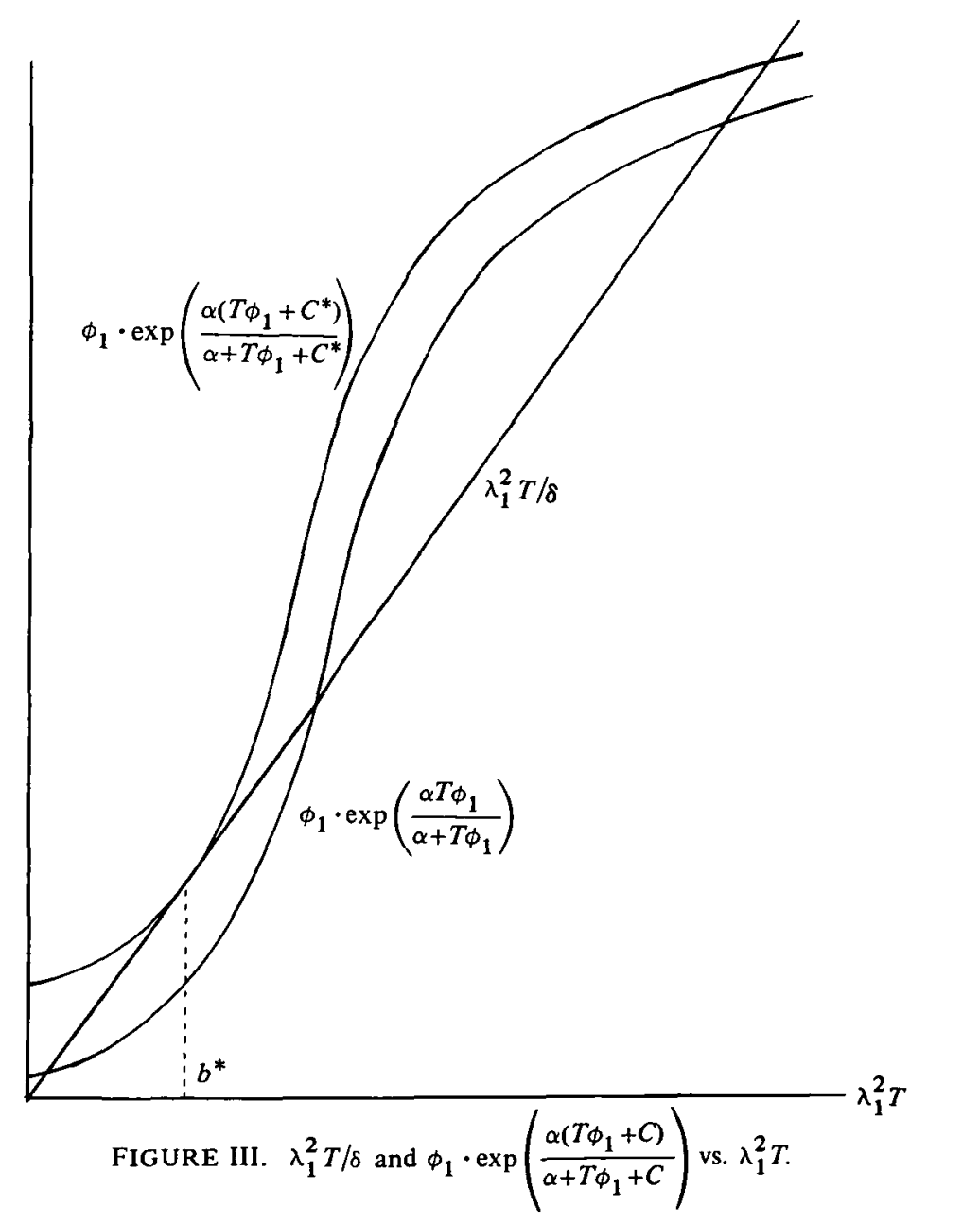


then it is clear from (4-7) and Figure III that there is only one equilibrium value for $T$, which is super-critical-in the sense that it is larger than the maximum of the intersections of $\lambda_{1}^{2} T / \delta$ and

$$
\varphi_{1} \cdot \exp \left[\frac{\alpha T \varphi_{1}+C^{*}}{\alpha+T \varphi_{1}+C^{*}}\right]
$$

Conversely, if

$$
\varphi_{1} \cdot \exp \left[\frac{\alpha\left(T \varphi_{1}+P(\xi, \infty)\right)}{\alpha+T \varphi_{1}+P(\xi, \infty)}\right]<\varphi_{1} \cdot \exp \left[\frac{\alpha\left(T \varphi_{1}+C^{*}\right)}{\alpha+T \varphi_{1}+C^{*}}\right],
$$

then the equilibrium value of $T$ will be sub-critical, - in the sense that it is less than $b^{*} / \lambda_{1}^{2}$. Thus, the number $C^{*}$ provides a demarkation value in assessing the influence of the boundary data $g(\mathbf{x}, t)$. Clearly, $C^{*}$ depends on $\delta$.

As an illustration, we consider the case of an infinite slab, $0<x<1$, with non-zero boundary conditions. The quantities $\lambda_{1}$ and $\varphi_{1}$ are known from previous studies to be $\lambda_{1}=\pi$ and $\varphi_{1}=\sqrt{2} \sin \pi x$. We take $\alpha=20, \delta=5.61 \times$ $10^{-3}$, and we compute $C^{*}$ to be 11.91 , and $b^{*}=25.5$. Now, if we take the non-zero boundary conditions to $\theta(0, t)=A$ and $\theta(1, t)=0$, we find that $P(x, \infty)=A(1-x)$. By varying the value of $A$, we see that the solution is sub-critical if $A<20$ and super-critical if $A>20$, a result agreeing quite well with that given in Tam and Chapman [13].

\section{IVb. Construction of upper and lower solutions}

Let $P$ and $T$ be as defined in (4-5) and equation (4-8). Let $m(\mathbf{x})>0$ be a function to be determined. We consider the IBVP

$$
\chi_{t}-\nabla^{2} \chi=\delta \exp \left[\frac{\alpha\left(m T \varphi_{1}+P\right)}{\alpha+m T \varphi_{1}+P}\right]
$$

subject to $\chi(\mathbf{x}, 0)=0 ; \chi(\mathbf{x}, t)=g(\mathbf{x}, t)>0, \mathbf{x} \in \partial D$. The solution of (4-9) and $(4-10)$ can be represented by (see [2], page 291)

$$
\begin{aligned}
\chi(x, t)= & \delta \int_{0}^{t} G(\mathbf{x}, \xi, t-\tau) \cdot \exp \left[\frac{\alpha\left(m T(\tau) \varphi_{1}(\xi)+P(\xi, \tau)\right)}{\alpha+m T(\tau) \Phi_{1}(\xi)+P(\xi, \tau)}\right] d \tau \\
& +P(\mathbf{x}, t) .
\end{aligned}
$$

As in section IIIb, we want to choose $m$ so that $\chi(x, t)$ is a lower solution of equations (1-1), (1-2) and (1-3), with $h(\mathbf{x})=0$. Thus, we are led to the requirement

$$
\begin{aligned}
m T(t) \varphi_{1}(x) \leqslant & \delta \int_{0}^{t} G(\mathbf{x}, \boldsymbol{\xi}, t-\tau) \\
& \cdot \exp \left[\frac{\alpha\left(m T(\tau) \varphi_{1}(\xi)+P(\xi, \tau)\right)}{\alpha+m T(\tau) \varphi_{1}(\xi)+P(\xi, \tau)}\right] d \tau .
\end{aligned}
$$


From equation (4-7) we have

$$
\begin{aligned}
m T(t) \varphi_{1}(x)= & \delta m \int_{0}^{t} \exp \left[-\lambda_{1}^{2}(t-\tau)\right] \varphi_{1}(\mathbf{x}) \varphi_{1}(\xi) \\
& \cdot \exp \left[\frac{\alpha\left(T(\tau) \varphi_{1}(\xi)+P(\xi, \tau)\right)}{\alpha+T(\tau) \varphi_{1}(\xi)+P(\xi, \tau)}\right] d \tau .
\end{aligned}
$$

Proceeding in exactly the same manner as in section IIIb, we see that a function $m(\mathbf{x})$ can be chosen to satisfy (4-12), thus making $\chi(x, t)$ a lower solution. In an analogous manner, and as in section IIIb, we can construct an upper solution. In particular, we can consider the solution of (4-8) and (4-9) with $m=1$ as a linearized approximate solution to the original problem.

\section{IVc. The combined influence of the initial and boundary conditions}

We now consider the full problem comprising equations (1-1), (1-2) and (1-3). It is clear that if either $h(\mathbf{x})$ or $g(\mathbf{x}, t)$ is such that one of the criteria obtained in Sections III and IV for super-criticality is met, the solution will be super-critical. The question therefore is: suppose taken separately, neither $h(\mathbf{x})$ nor $g(\mathbf{x}, t)$ causes super-criticality, will their combined effect be super-critical? To answer this, we let $S(\mathbf{x}, t)$ be the solution of IBVP

$$
\begin{gathered}
S_{t}-\nabla^{2} S=0, \\
S(\mathbf{x}, 0)=h(\mathbf{x}) ; \quad S(\mathbf{x}, t)=g(\mathbf{x}, t), \mathbf{x} \in \partial D .
\end{gathered}
$$

If we now let $\theta=\psi+S$, equations (1-1), (1-2) and (1-3) become

$$
\begin{gathered}
\psi_{t}-\nabla^{2} \psi=\delta \exp \left[\frac{\alpha(\psi+S)}{\alpha+\psi+S}\right], \\
\psi(\mathbf{x}, 0)=0 ; \quad \psi(\mathbf{x}, t)=0, \mathbf{x} \in \partial D,
\end{gathered}
$$

and the solution can be expressed in terms of an integral equation.

$$
\psi=\delta \int_{0}^{t} G(\mathbf{x}, \boldsymbol{\xi}, t-\tau) \cdot \exp \left[\frac{\alpha(\psi+S(\xi, \tau))}{\alpha+\psi+S(\xi, \tau)}\right] d \tau .
$$

Now, if at any time $t_{1}, S(x, t)$ evolves to such an extent that $(\psi+S)_{t_{1}}$ satisfies the sufficiency conditions as imposed on $h$ in III, then our previous results show that the solution of (1-1), (1-2) and (1-3) is super-critical. However, as remarked after (4-6), since we do not know $\psi$ at $t_{1}$, we can only impose the sufficiency condition for super-criticality on $S$.

\section{$V$. The influence of the Biot number in the case of general boundary conditions}

Instead of the boundary condition (1-3), a more general condition, taking into account Newtonian cooling at the surface, is

$$
\frac{\partial \theta}{\partial \nu}+\beta \theta=0, \quad x \in \partial D
$$


where $\nu$ is the outward normal direction. The parameter $\beta>0$ is referred to as the Biot number. The case $\beta \rightarrow \infty$ yields the homogeneous boundary condition $\theta=0$. Now, with $0<\beta<\infty$, the boundary temperature will not be fixed at zero. Consequently, the heat conduction away from the surface will be less pronounced, implying that more heat will be retained in the body. Thus, we expect the super-critical state will result with a smaller value of $\delta$, and/or lower initial temperature profile. Because of the relative ease of the required computation, we shall treat the case with non-homogeneous initial data via the integral-equation approach.

Let $\left\{x_{n}\right\}$ and $\left\{\mu_{n}^{2}\right\}$ be the normalized eigenfunctions and eigenvalues, respectively, of the BVP

$$
\begin{array}{ll}
\nabla^{2} \chi_{n}=-\mu_{n}^{2} \chi_{n}, & \mathrm{x} \in D, \\
\frac{\partial \chi_{n}}{\partial \nu}+\beta \chi_{n}=0, & \mathrm{x} \in \partial D,
\end{array}
$$

and let $K(\mathbf{x}, \xi, t)$ be the Robin function for the system (see page 289, [2])

$$
\begin{gathered}
K_{t}-\nabla^{2} K=0 \\
K(\mathbf{x}, 0)=0 ; \quad \frac{\partial K}{\partial \nu}+\beta K=0 ; \mathbf{x} \in \partial D .
\end{gathered}
$$

We have

$$
K(\mathbf{x}, \xi, t)=\sum_{n=1}^{\infty} \exp \left(-\mu_{n}^{2} t\right) \chi_{n}(\mathbf{x}) \chi_{n}(\xi) .
$$

Clearly, the eigenvalues now depend on the parameter $\beta$. We rewrite equations $(1-1),(1-2)$ and $(5-1)$ as the integral equation

$$
\theta(\mathbf{x}, t)=K(\mathbf{x}, \xi, t) \cdot h(\xi)+\delta \int_{0}^{t} K(\mathbf{x}, \xi, t-\tau) \cdot \exp \left\{\frac{\alpha \theta(\xi, \tau)}{\alpha+\theta(\xi, \tau)}\right\} d \tau
$$

Following our previous work, we can define an iteration process, and then use an asymptotic argument to assess the influence of both $h(\mathbf{x})$ and $\beta$. For the three simple geometries (Class $\mathrm{A}$ ), the respective eigenvalues and eigenfunctions are given as follows:

(a) Slab: $-1<x<1$

$$
\chi_{n}=\left(\frac{2 \mu_{n}}{2 \mu_{n}+\sin ^{2} \mu_{n}}\right)^{1 / 2} \cos \mu_{n} x
$$

where $\mu_{n}$ is determined from

$$
\mu_{n} \tan \mu_{n}=\beta \text {. }
$$


TABLE Ia. Values of $\delta_{e}, \delta_{\mathrm{cr}}$, and $v^{*}$

for some values of $\alpha$ and $\beta$ for the slab.

\begin{tabular}{|c|c|c|c|c|}
\hline & \multicolumn{2}{|l|}{$\beta=1.0$} & \multicolumn{2}{|l|}{$\beta=10$} \\
\hline \multirow{4}{*}{$\alpha=8$} & $\delta$ & $v^{*}$ & $\delta$ & $v^{*}$ \\
\hline & $\delta_{e}=3.75 \times 10^{-2}$ & 48.2 & $\delta_{e}=0.10535$ & 119.8 \\
\hline & 0.25 & 3.05 & 0.5 & 14 \\
\hline & $\delta_{\text {cr }}=0.314$ & 1.41 & $\delta_{c r}=0.855$ & 3.5 \\
\hline \multirow{3}{*}{$a=20$} & $\delta_{c}=1.5826 \times 10^{-6}$ & 372.2 & $\delta_{e}=4.47 \times 10^{-6}$ & 932 \\
\hline & 0.1 & 4.2 & 0.2 & 13.1 \\
\hline & $\delta_{\mathrm{cr}}=0.286$ & 1.2 & $\delta_{\mathrm{cr}}=0.775$ & 2.7 \\
\hline \multirow{3}{*}{$\alpha=40$} & $\delta_{e}=1.34 \times 10^{-14}$ & 1576 & $\delta_{e}=3.796 \times 10^{-14}$ & 3954.5 \\
\hline & 0.1 & 3.65 & 0.2 & 10 \\
\hline & $\delta_{\mathrm{cr}}=0.27027$ & 1.10 & $\delta_{\mathrm{cr}}=0.7519$ & 2.6 \\
\hline \multirow{3}{*}{$\alpha=100$} & \multicolumn{2}{|l|}{$\delta_{e}=$ overflow } & \multicolumn{2}{|l|}{$\delta_{e}=$ overflow } \\
\hline & 0.1 & 3.3 & 0.2 & 9.2 \\
\hline & $\delta_{\mathrm{cr}}=0.2703$ & 1.0 & $\delta_{\mathrm{cr}}=0.735$ & 2.55 \\
\hline
\end{tabular}

TABLE Ib. Values of $\delta_{e}, \delta_{\mathrm{cr}}$, and $v^{*}$ for some values of $\alpha$ and $\beta$ for the slab.

\begin{tabular}{|c|c|c|c|c|}
\hline & \multicolumn{2}{|l|}{$\beta=100$} & \multicolumn{2}{|c|}{$\beta=10000$} \\
\hline & $\delta$ & $v^{*}$ & $\delta$ & $v^{*}$ \\
\hline \multirow{3}{*}{$\alpha=8$} & $\delta_{e}=0.1247$ & 136 & $\delta_{e}=0.1274$ & 138.3 \\
\hline & 0.5 & 16 & 0.2 & 16.4 \\
\hline & $\delta_{\mathrm{cr}}=1.00$ & 4.0 & $\delta_{\mathrm{cr}}=1.0204$ & 4.0 \\
\hline \multirow{3}{*}{$\alpha=20$} & $\delta_{e}=5.293 \times 10^{-6}$ & 1058 & $\delta_{e}=5.4085 \times 10^{-6}$ & 1075 \\
\hline & 0.2 & 14.6 & 0.2 & 14.8 \\
\hline & $\delta_{\mathrm{cr}}=0.9174$ & 3.1 & $\delta_{\mathrm{cr}}=0.926$ & 3.3 \\
\hline \multirow{3}{*}{$\alpha=40$} & $\delta_{e}=4.494 \times 10^{-14}$ & 4488 & $\delta_{e}=4.592 \times 10^{-14}$ & 4561 \\
\hline & 0.2 & 12.1 & 0.2 & 13.7 \\
\hline & $\delta_{\mathrm{cr}}=0.885$ & 3.05 & $\delta_{\mathrm{cr}}=0.910$ & 3.1 \\
\hline \multirow{3}{*}{$\alpha=100$} & \multicolumn{2}{|l|}{$\delta_{e}$ overflow } & \multicolumn{2}{|l|}{$\delta_{e}$ overflow } \\
\hline & 0.2 & 11.1 & 0.2 & 11.4 \\
\hline & $\delta_{\mathrm{cr}}=0.8696$ & 3.0 & $\delta_{c r}=0.885$ & 3.0 \\
\hline
\end{tabular}


TABLE IIa. Values of $\delta_{e}, \delta_{\mathrm{cr}}$, and $v^{*}$

for some values of $\alpha$ and $\beta$ for the sphere.

\begin{tabular}{|c|c|c|c|c|}
\hline \multirow{4}{*}{$\alpha=8$} & $\delta$ & $v^{*}$ & $\delta$ & $v^{*}$ \\
\cline { 2 - 5 } & $\delta_{e}=0.1252$ & 232.6 & $\delta_{e}=0.42986$ & 597.4 \\
\cline { 2 - 5 } & $\delta=0.8333$ & 15 & $\delta=1.67$ & 65.5 \\
\cline { 2 - 5 } & $\delta_{\mathrm{cr}}=1.036$ & 7.3 & $\delta_{\mathrm{cr}}=3.265$ & 16.8 \\
\hline \multirow{3}{*}{$\alpha=20$} & $\delta_{e}=5.28 \times 10^{-6}$ & 1796 & $\delta_{e}=1.8446 \times 10^{-5}$ & 4732.8 \\
\cline { 2 - 5 } & $\delta=0.5$ & 15.4 & $\delta=0.5$ & 64 \\
\cline { 2 - 5 } & $\delta_{\mathrm{cr}}=0.952$ & 5.5 & $\delta_{\mathrm{cr}}=2.91$ & 14.2 \\
\hline \multirow{3}{*}{$\alpha=40$} & $\delta_{e}=4.4746 \times 10^{-14}$ & 7609 & $\delta_{e}=1.571 \times 10^{-13}$ & 20170 \\
\cline { 2 - 5 } & $\delta=0.5$ & 13.7 & $\delta=0.67$ & 47 \\
\cline { 2 - 5 } & $\delta_{\mathrm{cr}}=0.93$ & 5.1 & $\delta_{\mathrm{cr}}=2.857$ & 13.8 \\
\hline \multirow{3}{*}{$\alpha=100$} & $\delta_{e}$ overflow & & $\delta_{e}$ overflow & 12.6 \\
\cline { 2 - 5 } & $\delta=0.5$ & 12.9 & $\delta=067$ & \multicolumn{2}{c|}{$\delta$} \\
\cline { 2 - 5 } & $\delta_{\mathrm{cr}}=0.92$ & 5.0 & $\delta_{\mathrm{cr}}=2.759$ & \multicolumn{2}{c}{$\beta$} \\
\hline
\end{tabular}

TABLE IIb. Values of $\delta_{e}, \delta_{\mathrm{cr}}$ and $v^{*}$

for some values of $\alpha$ and $\beta$ for the sphere.

\begin{tabular}{|c|c|c|c|c|}
\hline & \multicolumn{2}{|l|}{$\beta=100$} & \multicolumn{2}{|c|}{$\beta=10000$} \\
\hline \multirow{4}{*}{$\alpha=8$} & $\delta$ & $v^{*}$ & $\delta$ & $v^{*}$ \\
\hline & $\delta_{e}=0.519$ & 629.2 & $\delta_{e}=0.5295$ & 632.4 \\
\hline & $\delta=2$ & 70 & $\delta=2$ & 71.5 \\
\hline & $\delta_{c r}=3.846$ & 18.75 & $\delta_{\mathrm{cr}}=3.922$ & 19.5 \\
\hline \multirow{3}{*}{$\alpha=20$} & $\delta_{e}=2.23 \times 10^{-5}$ & 4980.4 & $\delta_{e}=2.275 \times 10^{-5}$ & 5005.2 \\
\hline & $\delta=2$ & 317 & $\delta=2$ & 37.2 \\
\hline & $\delta_{\text {cr }}=3.478$ & 14.4 & $\delta_{\mathrm{cr}}=3.555$ & 15.5 \\
\hline \multirow{3}{*}{$\alpha=40$} & $\delta_{e}=1.8996 \times 10^{-13}$ & 21216 & $\delta_{e}=1.9379 \times 10^{-13}$ & 21321.8 \\
\hline & $\delta=2$ & 32 & $\delta=2$ & 32.2 \\
\hline & $\delta_{c r}=3.404$ & 14.0 & $\delta_{\mathrm{cr}}=3.478$ & 13.8 \\
\hline \multirow{3}{*}{$a=100$} & \multicolumn{2}{|l|}{$\delta_{e}$ overflow } & \multicolumn{2}{|l|}{$\delta_{\mathrm{e}}$ overflow } \\
\hline & $\delta=2$ & 29.5 & $\delta=2$ & 30 \\
\hline & $\delta_{\mathrm{cr}}=3.265$ & 13.9 & $\delta_{\mathrm{cr}}=3.333$ & 13.2 \\
\hline
\end{tabular}


TABLE IIIa. Values of $\delta_{e}, \delta_{\mathrm{cr}}$ and $v^{*}$ for some values of $\alpha$ and $\beta$ for the cylinder.

\begin{tabular}{|c|c|c|c|c|}
\hline & \multicolumn{2}{|l|}{$\beta=1.0$} & \multicolumn{2}{|l|}{$\beta=10$} \\
\hline & $\delta$ & $v^{*}$ & 8 & $v^{*}$ \\
\hline \multirow{3}{*}{$\alpha=8$} & $\delta_{e}=0.1687$ & 241.6 & $\delta_{e}=0.285$ & 345 \\
\hline & 0.333 & 15 & $\delta=1$ & 26 \\
\hline & $\delta_{\mathrm{cr}}=0.676$ & 4.0 & $\delta_{\mathrm{cr}}=1.951$ & 10 \\
\hline \multirow{3}{*}{$\alpha=20$} & $\delta_{e}=7.1482 \times 10^{-6}$ & 1882 & $\delta_{e}=1.218 \times 10^{-5}$ & 2711 \\
\hline & 0.2 & 8.1 & $\delta=0.5$ & 28.3 \\
\hline & $\delta_{\mathrm{cr}}=0.606$ & 3.1 & $\delta_{\mathrm{cr}}=1.768$ & 7.7 \\
\hline \multirow{3}{*}{$\alpha=40$} & $\delta_{e}=6.066 \times 10^{-14}$ & 7991.7 & $\delta_{e}=1.0358 \times 10^{-13}$ & 11529 \\
\hline & 0.2 & 9.8 & $\delta=0.25$ & 24.3 \\
\hline & $\delta_{\mathrm{cr}}=0.592$ & 3.0 & $\delta_{\mathrm{cr}}=1.728$ & 7.3 \\
\hline \multirow{3}{*}{$\alpha=100$} & \multicolumn{2}{|l|}{$\delta_{e}$ overflow } & \multicolumn{2}{|l|}{$\delta_{e}$ overflow } \\
\hline & $\delta=0.2$ & 5.9 & $\delta=0.25$ & 22.4 \\
\hline & $\delta_{\mathrm{cr}}=0.588$ & 2.7 & $\delta_{\mathrm{cr}}=1.684$ & 7.2 \\
\hline
\end{tabular}

TABLE IIIb. Values of $\delta_{e}, \delta_{\mathrm{cr}}$ and $v^{*}$ for some values of $\alpha$ and $\beta$ for the cylinder.

\begin{tabular}{|c|c|c|c|c|}
\hline & \multicolumn{2}{|l|}{$\beta=100$} & \multicolumn{2}{|c|}{$\beta=10000$} \\
\hline & $\delta$ & $v^{*}$ & $\delta$ & $v^{*}$ \\
\hline \multirow{3}{*}{$\alpha=8$} & $\delta_{e}=0.30274$ & 356 & $\delta_{e}=0.305$ & 357 \\
\hline & $\delta=0.833$ & 57.5 & 0.986 & 50.7 \\
\hline & $\delta_{\mathrm{cr}}=2.286$ & 11.0 & $\delta_{c r}=2.350$ & 10.6 \\
\hline \multirow{3}{*}{$\alpha=20$} & $\delta_{e}=1.2934 \times 10^{-5}$ & 2795 & $\delta_{e}=1.30 \times 10^{-5}$ & 2804 \\
\hline & $\delta=0.4$ & 38 & .706 & 42.5 \\
\hline & $\delta_{\mathrm{cr}}=2.051$ & 8.5 & $\delta_{\mathrm{cr}}=2.1086$ & 8.5 \\
\hline \multirow{3}{*}{$\alpha=40$} & $\delta_{e}=1.10 \times 10^{-13}$ & 11884 & $\delta_{e}=1.107 \times 10^{-13}$ & 11922 \\
\hline & $\delta=0.667$ & 26.2 & 0.189 & 40 \\
\hline & $\delta_{\mathrm{cr}}=2.025$ & 8.0 & $\delta_{\mathrm{cr}}=2.055$ & 7.96 \\
\hline \multirow{3}{*}{$\alpha=100$} & \multicolumn{2}{|l|}{$\delta_{e}$ overflow } & \multicolumn{2}{|l|}{$\delta_{e}$ overflow } \\
\hline & $\delta=0.667$ & 23.8 & 0.1130 & 40 \\
\hline & $\delta_{\mathrm{cr}}=1.975$ & 7.5 & $\delta_{\mathrm{cr}}=2.01926$ & 7.697 \\
\hline
\end{tabular}


TABLE IV. Comparison of $\alpha_{\mathrm{tr}}$ and $\delta_{\mathrm{tr}}$ with results from Fenaughty et al.

\begin{tabular}{|l|l|l|l|l|l|l|l|}
\hline \multirow{2}{*}{$\begin{array}{l}\text { No. } \\
\text { Not }\end{array}$} & & \multicolumn{2}{|c|}{ Slab } & \multicolumn{2}{c|}{ Cylinder } & \multicolumn{2}{c|}{ Sphere } \\
\cline { 3 - 8 } & & $\begin{array}{l}\text { present } \\
\text { result }\end{array}$ & $\begin{array}{l}\text { Fenaughty } \\
\text { et al. }\end{array}$ & $\begin{array}{l}\text { present } \\
\text { result }\end{array}$ & $\begin{array}{l}\text { Fenaughty } \\
\text { et al. }\end{array}$ & $\begin{array}{l}\text { present } \\
\text { result }\end{array}$ & $\begin{array}{l}\text { Fenaughty } \\
\text { et al. }\end{array}$ \\
\hline \multirow{3}{*}{$\beta=0.01$} & $\alpha_{\mathrm{tr}}$ & 4.00004 & 4 & 4.0015 & 4 & 4.000002 & 4 \\
\cline { 2 - 8 } & $\delta_{\mathrm{tr}}$ & 0.005395 & 0.00542 & 0.03038 & 0.01085 & 0.016273 & 0.01629 \\
\hline \multirow{3}{*}{$\beta=0.1$} & $\alpha_{\mathrm{tr}}$ & 4.00023 & 4.00016 & 4.0015 & 4.00016 & 4.00017 & 4.00016 \\
\cline { 2 - 8 }$\beta=1$ & $\delta_{\mathrm{tr}}$ & 0.052359 & 0.05262 & 0.323925 & 0.10609 & 0.1591718 & 0.15992 \\
\hline \multirow{3}{*}{$\beta=10$} & $\alpha_{\mathrm{tr}}$ & 4.01242 & 4.01220 & 4.0558 & 4.01494 & 4.01469 & 4.01445 \\
\cline { 2 - 8 } & $\delta_{\mathrm{tr}}$ & 0.399377 & 0.40049 & 1.734455 & 0.85234 & 1.33080 & 1.33340 \\
\hline \multirow{3}{*}{$\beta=100$} & $\alpha_{\mathrm{tr}}$ & 4.0642 & 4.06306 & 4.12352 & 4.11709 & 4.16843 & 4.16406 \\
\cline { 2 - 8 } & $\delta_{\mathrm{tr}}$ & 1.083952 & 1.08454 & 2.826147 & 248202 & 4.14390 & 4.14461 \\
\hline \multirow{3}{*}{$\beta=10000$} & $\alpha_{\mathrm{tr}}$ & 4.069395 & 4.06851 & 4.12833 & 4.130013 & 4.19165 & 4.18708 \\
\cline { 2 - 8 } & $\delta_{\mathrm{tr}}$ & 1.278937 & 1.28168 & 2.991818 & 2.94682 & 493478 & 4.94085 \\
\cline { 2 - 8 } & $\delta_{\mathrm{tr}}$ & 1.306765 & 1.30709 & 3.00997 & 3.00557 & 5.03346 & 5.03980 \\
\hline
\end{tabular}

(b) infinite cylinder: $0<r<1$

$$
\chi_{n}=\frac{\mu_{n}}{\sqrt{\pi\left(\mu_{n}^{2}+\beta^{2}\right)} J_{0}\left(\mu_{n}\right)} J_{0}\left(\mu_{n} r\right)
$$

where $\mu_{n}$ is determined from

$$
\beta J_{0}\left(\mu_{n}\right)=\mu_{n} J_{1}\left(\mu_{n}\right)
$$

(c) sphere: $0<r<1$

$$
\chi_{n}=\left[\frac{\mu_{n}}{\pi\left(2 \mu_{n}-\sin k^{2} \mu_{n}\right)}\right]^{1 / 2} \frac{\sin \mu_{n} r}{r}
$$

where $\mu_{n}$ is determined from

$$
\begin{gathered}
\cos \mu_{n}=0 ; \quad \beta=1, \\
\tan \mu_{n}=\frac{1}{1-\beta} \mu_{n}, \quad \beta \neq 1 .
\end{gathered}
$$

Representative computations for $\delta_{\mathrm{cr}}, \delta_{e}$, and $v^{*}$ for some values of $\beta$ and $\alpha$ for these cases are presented in Tables I, II and III. The numbers in these three tables are obtained graphically. In Table IV, we list the transitional values of $\alpha$, with the corresponding $\delta_{\mathrm{tr}}$, for the three class $A$ geometries. These numbers are determined numerically on an IBM personal computer in an iteractive manner. The 
corresponding values obtained by Fenaughty et al. [3] are listed for comparison. The agreement is good except for the values of $\delta_{\mathrm{tr}}$ for the cylinder for $\beta<10$. We recall the transitional value $\alpha_{\mathrm{tr}}$ is the value of a below which the time-independent version of equations (1-1), (1-3) has no multiple solutions, and $\delta_{\mathrm{tr}}$ is the limit of $\delta_{\mathrm{cr}}$ as $\alpha$ approaches $\alpha_{\mathrm{tr}}$ from above.

\section{Concluding remarks}

We have studied a nonlinear parabolic equation arising from the theory of autocatalytic reactions. For the case with homogeneous initial and Dirichlet boundary conditions, and when the parameter $\alpha$ is sufficiently large, we constructed upper and lower solutions from which definitive bounds on the critical value of $\delta$ are obtained. The role of the first eigenfunction is emphasized. We then consider the influence of the initial data. A two-step linearization method is used to demonstrate a procedure for the construction of upper and lower solutions, from which an approximate solution is obtained. The essence of the two-step method is that the dependence of the solution of the original problem on initial data and parameters can be inferred from the dependence of the solution of an associated ordinary differential equation on those quantities. Sufficient conditions for the solution of the ordinary differential equation to become super-critical, and hence the solution of the original nonlinear parabolic equation to become sub- or super-critical are obtained. Results obtained previously from an asymptotic method are shown to be consistent with the current results. Since the case with non-zero initial data includes the case with homogeneous initial data as a special case, the approximate evaluation of $\delta_{\mathrm{cr}}$ can be checked against the definitive bounds obtained in II. The influence of the boundary condition is considered next and tied in with the influence of the initial data. Lastly, we introduce a new parameter, the Biot number, in the form of a Robin boundary condition, and consider its influence of the solution. Values of $\delta_{e}, \delta_{\mathrm{cr}}, v^{*}$ and $\alpha_{\mathrm{cr}}$ as given by the asymptotic analysis are computed for a wide range of the Biot number, for the class $A$ geometries.

Of some interest is the two-step linearization method introduced in III. It is a capitalization of the recognition that the first eigenfunction associated with the linear problem plays a significant role in the nonlinear problem. Indeed, the derivation of the ordinary differential equation is based on what we have learned about the behaviour of the given parabolic equation, and is in the sense a culmination of our past efforts. The idea of tackling a difficult problem in two steps is not a new one, and conceivably this method may be used in other situations. 


\section{Acknowledgement}

The author is grateful to Drs. A. McNabb and G. Wake for helpful comments and discussions in the past few years, and for critical remarks that led to substantial improvements in the present revised version of this work. The author also thanks Mr. K. Chan and Miss J. Adams for doing some of the computations. Continuing support from the Natural Science and Engineering Research Council of Canada under grant A-5228 is acknowledged.

\section{References}

[1] C. Y. Chan, "A first initial boundary value problem for a semi-linear heat equation", SIAM J. Appl. Math. 22 (1972), 529-537.

[2] G. F. D. Duff and D. Naylor, Differential equations of applied mathematıcs (Wiley, 1966).

[3] K. F. Fenaughty, A. A. Lacey and G. C. Wake, "The disappearance of criticality for small activation energy with arbitrary Biot number", Combustion and Flame 45 (1982), 287-291.

[4] D. A. Frank-Kamenetskii, Diffuston and heat transfer in chemical kinetics (Translation editor J. P. Appleton), (Plenum Press, New York, 1959).

[5] W. Kordylewski, "Degenerate critical points of thermal explosion", Komunikat $I-20 / K-020 / 78$, (Technical University of Wroclaw 1978).

[6] J. R. Parks, "Criticality criteria for various configurations of a self-heating chemical as functions of activation energy and temperature of assembly", J. Chem. Phys. 34 (1961), 46-50.

[7] D. H. Sattinger, "A nonlinear parabolic system in the theory of combustion", Quart. Appl. Math. (1975), 47-61.

[8] K. K. Tam, "Construction of upper and lower solutions for a problem in combustion theory", J. Math. Anal. Appl. 69 (1979), 131-145.

[9] K. K. Tam, "Initial data and criticality for a problem in combustion theory", J. Math. Anal. Appl. 77 (1980), 626-634.

[10] K. K. Tam, "On the influence of the initial data in a combustion problem", J. Austral. Math. Soc. Ser. B 22 (1980), 193-209.

[11] K. K. Tam, "Computation of critical parameters for a problem in combustion theory," $J$. Austral. Math. Soc. Ser. B 24 (1982), 40-46.

[12] K. K. Tam, "On the Oseen linearization of the swirling flow boundary layer", J. Fluid Mech. 75 (1976), 777-790.

[13] K. K. Tam and P. B. Chapman, "Thermal ignition in a reactive slab with unsymmetric boundary temperatures", J. Austral. Math. Soc. Ser. B 24 (1983), 279-288.

[14] K. K. Tam and M. T. Kiang, "The response to a hot spot in a combustion problem," $J$. Austral. Math. Soc. Ser. B 23 (1981), 95-102. 